PROCEEDINGS OF THE

AMERICAN MATHEMATICAL SOCIETY

Volume 138, Number 2, February 2010, Pages 505-516

S 0002-9939(09)10096-5

Article electronically published on October 6, 2009

\title{
SUM OF MULTIPLE $q$-ZETA VALUES
}

\author{
ZHONG-HUA LI
}

(Communicated by Ken Ono)

\begin{abstract}
The generating function of the sums of multiple $q$-zeta values with fixed weights, depths and 1-heights, 2 -heights, $\ldots, r$-heights is represented in terms of specializations of basic hypergeometric functions.
\end{abstract}

\section{INTRODUCTION}

In this paper, let $\mathbf{k}=\left(k_{1}, k_{2}, \ldots, k_{n}\right)$ be a sequence of positive integers. For such a $\mathbf{k}=\left(k_{1}, k_{2}, \ldots, k_{n}\right)$, as in [4, we define the weight, depth and $i$-height of $\mathbf{k}$ by $\operatorname{wt}(\mathbf{k})=k_{1}+k_{2}+\cdots+k_{n}, \operatorname{dep}(\mathbf{k})=n$ and $i$-ht $(\mathbf{k})=\#\left\{l \mid k_{l}>i\right\}$, respectively. A sequence $\mathbf{k}=\left(k_{1}, k_{2}, \ldots, k_{n}\right)$ is admissible if $k_{1}>1$.

Throughout this paper, we assume that $q$ is a real number with $0<q<1$. For a positive integer $n$, its $q$-analogue is defined by

$$
[n]=[n]_{q}=1+q+q^{2}+\cdots+q^{n-1}=\frac{1-q^{n}}{1-q} .
$$

For a sequence $\mathbf{k}=\left(k_{1}, k_{2}, \ldots, k_{n}\right)$, the multiple $q$-zeta value $\zeta[\mathbf{k}]$ is defined by the following $q$-series (see [1, 8]):

$$
\zeta[\mathbf{k}]=\sum_{m_{1}>m_{2}>\cdots>m_{n}>0} \frac{q^{m_{1}\left(k_{1}-1\right)+m_{2}\left(k_{2}-1\right)+\cdots+m_{n}\left(k_{n}-1\right)}}{\left[m_{1}\right]^{k_{1}}\left[m_{2}\right]^{k_{2}} \cdots\left[m_{n}\right]^{k_{n}}} .
$$

If $\mathbf{k}$ is admissible, the right hand side of (1.1) absolutely converges. Taking the limit $q \rightarrow 1^{-}$, we obtain the usual multiple zeta value $\zeta(\mathbf{k})$ :

$$
\lim _{q \rightarrow 1^{-}} \zeta[\mathbf{k}]=\zeta(\mathbf{k})=\sum_{m_{1}>m_{2}>\cdots>m_{n}>0} \frac{1}{m_{1}^{k_{1}} m_{2}^{k_{2}} \cdots m_{n}^{k_{n}}} .
$$

As in [6], for a sequence $\mathbf{k}=\left(k_{1}, k_{2}, \ldots, k_{n}\right)$, the multiple $q$-polylogarithms (of one variable) $\mathrm{Li}_{\mathbf{k}}[t]$ are defined by

$$
\operatorname{Li}_{\mathbf{k}}[t]=\operatorname{Li}_{k_{1}, k_{2}, \ldots, k_{n}}[t]=\sum_{m_{1}>m_{2}>\cdots>m_{n}>0} \frac{t^{m_{1}}}{\left[m_{1}\right]^{k_{1}}\left[m_{2}\right]^{k_{2}} \cdots\left[m_{n}\right]^{k_{n}}} .
$$

Received by the editors March 1, 2009, and, in revised form, June 20, 2009.

2000 Mathematics Subject Classification. Primary 11M41, 11M99.

Key words and phrases. Multiple $q$-zeta values, multiple $q$-polylogarithms, basic hypergeometric functions.

(C)2009 American Mathematical Society Reverts to public domain 28 years from publication 
The right hand side of (1.2) is absolutely convergent for $|t|<1$. Taking the limit $q \rightarrow 1^{-}$, we get the multiple polylogarithms (of one variable)

$$
\lim _{q \rightarrow 1^{-}} \operatorname{Li}_{\mathbf{k}}[t]=\sum_{m_{1}>m_{2}>\cdots>m_{n}>0} \frac{t^{m_{1}}}{m_{1}^{k_{1}} m_{2}^{k_{2}} \cdots m_{n}^{k_{n}}} .
$$

Ohno and Zagier [5] studied the generating function of the sums of multiple zeta values with fixed weights, depths and 1-heights. They proved that these sums can be written as polynomials of Riemann zeta values with rational coefficients. We studied the generating function of the sums of multiple zeta values with fixed weights, depths and 1-heights, 2-heights, $\ldots, r$-heights for any natural number $r$ in 4. We found that this generating function can be represented by specializations of the generalized hypergeometric function ${ }_{r+1} F_{r}$. In [1, Bradley partially gave a $q$-analogue of the result of Ohno and Zagier [5], and in [6], Okuda and Takeyama generalized this result to multiple $q$-zeta values completely.

In this paper, we give a $q$-analogue of our results of [4, which generalizes the result of Okuda and Takeyama in [6]. We represent the generating function of the sums of multiple $q$-zeta values with fixed weights, depths and 1-heights, 2-heights, $\ldots, r$-heights in terms of specializations of basic hypergeometric functions. In Section 2, we list some preliminary facts related to basic hypergeometric functions. In Section 3, after treating the case of the sum of multiple $q$-polylogarithms, we obtain our main theorem (Theorem 3.2) about the sum of multiple $q$-zeta values.

The author thanks J. Okuda for encouragement and suggestions and the referee for many useful suggestions.

\section{2. $q$-ANALOGUES}

Let us recall the definition of basic hypergeometric functions from the book 2 . The $q$-shifted factorial $(a ; q)_{n}$ is defined by

$$
(a ; q)_{n}= \begin{cases}1, & n=0, \\ (1-a)(1-a q) \cdots\left(1-a q^{n-1}\right), & n=1,2, \ldots\end{cases}
$$

To simplify the notation, we denote the products $\left(a_{1} ; q\right)_{n}\left(a_{2} ; q\right)_{n} \cdots\left(a_{m} ; q\right)_{n}$ by $\left(a_{1}, a_{2}, \ldots, a_{m} ; q\right)_{n}$. For a natural number $r$, the basic hypergeometric series is defined by

$$
{ }_{r+1} \phi_{r}\left[\begin{array}{c}
a_{1}, a_{2}, \ldots, a_{r+1} \\
b_{1}, \ldots, b_{r}
\end{array} ;, t\right]=\sum_{n=0}^{\infty} \frac{\left(a_{1}, a_{2}, \ldots, a_{r+1} ; q\right)_{n}}{\left(q, b_{1}, \ldots, b_{r} ; q\right)_{n}} t^{n},
$$

where $b_{j} \neq q^{-m}$ for $m=0,1, \ldots$ and $j=1,2, \ldots, r$. The right hand side of (2.1) is absolutely convergent for $|t|<1$, which gives the basic hypergeometric function.

The $q$-Stirling numbers $S_{q}(n, k)$ of the second kind are defined by the following recurrence $(3,7])$ :

$$
S_{q}(n, k)= \begin{cases}q^{k-1} S_{q}(n-1, k-1)+[k] S_{q}(n-1, k), & \text { if } 0<k \leq n, \\ 1, & \text { if } n=k=0, \\ 0, & \text { otherwise. }\end{cases}
$$

As in [2, Exercise 1.12], let $\mathcal{D}_{q}$ be the $q$-difference operator

$$
\left(\mathcal{D}_{q} f\right)(t)=\frac{f(t)-f(q t)}{(1-q) t} .
$$

Let $\mathcal{D}_{q}^{n} u=\mathcal{D}_{q}\left(\mathcal{D}_{q}^{n-1} u\right)$ and $\mathcal{D}_{q}^{0} u=u$. 
Lemma 2.1. For a nonnegative integer $n$, we have

$$
\begin{aligned}
& \mathcal{D}_{q r+1}^{n} \phi_{r}\left[\begin{array}{c}
a_{1}, a_{2}, \ldots, a_{r+1} ; q, a t \\
b_{1}, \ldots, b_{r}
\end{array}\right] \\
& =\frac{\left(a_{1}, a_{2}, \ldots, a_{r+1} ; q\right)_{n} a^{n}}{\left(b_{1}, \ldots, b_{r} ; q\right)_{n}(1-q)^{n}}{ }_{r+1} \phi_{r}\left[\begin{array}{c}
a_{1} q^{n}, a_{2} q^{n}, \ldots, a_{r+1} q^{n} \\
b_{1} q^{n}, \ldots, b_{r} q^{n}
\end{array} q, a t\right] .
\end{aligned}
$$

One can prove the above lemma by using induction on $n$.

We define another $q$-difference operator $\Theta_{q}$ by $\Theta_{q}=t \mathcal{D}_{q}$. In other words, we have

$$
\left(\Theta_{q} f\right)(t)=\frac{f(t)-f(q t)}{1-q} .
$$

Let $\Theta_{q}^{n} u=\Theta_{q}\left(\Theta_{q}^{n-1} u\right)$ and $\Theta_{q}^{0} u=u$.

By using induction and the recurrence of $q$-Stirling numbers of the second kind, we get the following fact.

Lemma 2.2. For a nonnegative integer $n$, we have

$$
\Theta_{q}^{n}=\sum_{m=0}^{n} S_{q}(n, m) t^{m} \mathcal{D}_{q}^{m} .
$$

Finally, following [2, Exercise 1.31], we have:

Lemma 2.3. Let $r$ be a positive integer and $a_{1} \cdots a_{r+1} b_{1} \cdots b_{r} \neq 0$. Let $b_{j} \neq q^{-m}$ for $m=0,1, \ldots$ and $j=1,2, \ldots, r$. Set $v(t)={ }_{r+1} \phi_{r}\left[\begin{array}{c}a_{1}, a_{2}, \ldots, a_{r+1} \\ b_{1}, \ldots, b_{r}\end{array} q, \frac{b_{1} \cdots b_{r}}{a_{1} \cdots a_{r+1} q^{r}} t\right]$. We have that $v(t)$ satisfies the following q-difference equation:

$$
\begin{aligned}
& \Theta_{q}\left(\Theta_{q}+\frac{q-b_{1}}{b_{1}(1-q)}\right) \cdots\left(\Theta_{q}+\frac{q-b_{r}}{b_{r}(1-q)}\right) v(t) \\
= & t\left(\Theta_{q}+\frac{1-a_{1}}{a_{1}(1-q)}\right) \cdots\left(\Theta_{q}+\frac{1-a_{r+1}}{a_{r+1}(1-q)}\right) v(t) .
\end{aligned}
$$

\section{MAIN RESULTS}

3.1. Main theorems. We abbreviate $1-q$ as $q_{1}$. Let $r$ be a fixed positive integer. For given nonnegative integers $k, n, h_{1}, h_{2}, \ldots, h_{r}$, we define $I_{j}\left(k, n, h_{1}, h_{2}, \ldots, h_{r}\right)$ as a set

$$
\left\{\mathbf{k}=\left(k_{1}, \ldots, k_{n}\right) \mid \operatorname{wt}(\mathbf{k})=k, \operatorname{dep}(\mathbf{k})=n, 1-\mathrm{ht}(\mathbf{k})=h_{1}, \ldots, r-\mathrm{ht}(\mathbf{k})=h_{r}, k_{1} \geq j+2\right\}
$$

and

$$
G_{j}\left(k, n, h_{1}, h_{2}, \ldots, h_{r} ; t\right)=\sum_{\mathbf{k} \in I_{j}\left(k, n, h_{1}, h_{2}, \ldots, h_{r}\right)} \operatorname{Li}_{\mathbf{k}}[t],
$$

where $j=-1,0, \ldots, r-1$. In the above definition, the sum is assigned to be zero if $I_{j}\left(k, n, h_{1}, h_{2}, \ldots, h_{r}\right)$ is empty and $G_{-1}(\underbrace{0,0, \ldots, 0}_{r+2} ; t)=1$. For $j=0,1, \ldots, r-1$, we also study the sum $G_{j}\left(k, n, h_{1}, \ldots, h_{r}\right)$ of multiple $q$-zeta values, where

$$
G_{j}\left(k, n, h_{1}, \ldots, h_{r}\right)=\sum_{\mathbf{k} \in I_{j}\left(k, n, h_{1}, \ldots, h_{r}\right)} \zeta[\mathbf{k}] .
$$


We define the following generating functions:

$$
\begin{gathered}
\Phi_{j}\left(x_{1}, x_{2}, \ldots, x_{r+2} ; t\right)=\sum_{k, n, h_{1}, h_{2}, \ldots, h_{r} \geq 0} G_{j}\left(k, n, h_{1}, h_{2}, \ldots, h_{r} ; t\right) \\
\cdot x_{1}^{k-n-\sum_{j=1}^{r} h_{j}} x_{2}^{n-h_{1}} x_{3}^{h_{1}-h_{2}} x_{4}^{h_{2}-h_{3}} \cdots x_{r+1}^{h_{r-1}-h_{r}} x_{r+2}^{h_{r}}
\end{gathered}
$$

and

$$
\begin{gathered}
\Psi_{j}\left(u_{1}, u_{2}, \ldots, u_{r+2}\right)=\sum_{k, n, h_{1}, \ldots, h_{r} \geq 0} G_{j}\left(k, n, h_{1}, \ldots, h_{r}\right) \\
\cdot u_{1}^{k-n-\sum_{j=1}^{r} h_{j}} u_{2}^{n-h_{1}} u_{3}^{h_{1}-h_{2}} \cdots u_{r+1}^{h_{r-1}-h_{r}} u_{r+2}^{h_{r}} .
\end{gathered}
$$

Here $x_{1}, \ldots, x_{r+2}$ and $u_{1}, \ldots, u_{r+2}$ are variables with relations

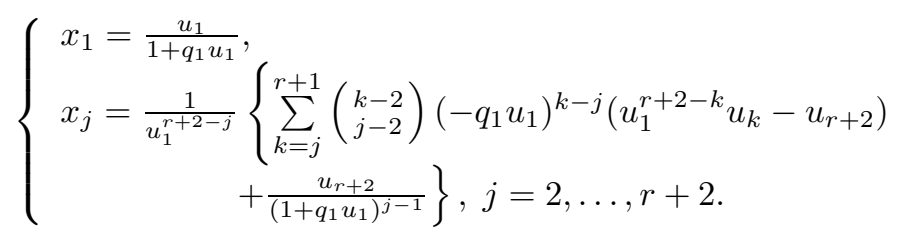

It is easy to show that (3.1) is equivalent to

$$
\left\{\begin{array}{c}
u_{1}=\frac{x_{1}}{1-q_{1} x_{1}}, \\
u_{j}=\frac{1}{x_{1}^{r+2-j}}\left\{\sum_{i=j-1}^{r}\left(\begin{array}{c}
i-1 \\
j-2
\end{array}\right)\left(q_{1} x_{1}\right)^{i-j+1}\left(x_{1}^{r+1-i} x_{i+1}-x_{r+2}\right)\right. \\
\left.+\frac{x_{r+2}}{\left(1-q_{1} x_{1}\right)^{j-1}}\right\}, j=2, \ldots, r+2 .
\end{array}\right.
$$

Let us denote $\Phi_{j}=\Phi_{j}\left(x_{1}, x_{2}, \ldots, x_{r+2} ; t\right)$ for short. We represent these generating functions via basic hypergeometric functions in the following theorem.

Theorem 3.1. For any integer $j$ with $-1 \leq j \leq r-1$, we have

$$
\begin{aligned}
\Phi_{j}= & \frac{1}{x_{r+2}-x_{1} x_{r+1}}\left\{\sum_{i=0}^{r-1-j} A_{i}^{(j)} B_{i} t^{i}\right. \\
& \left.\times{ }_{r+1} \phi_{r}\left[\begin{array}{c}
a_{1} q^{i}, \ldots, a_{r+1} q^{i} \\
b q^{i}, \underbrace{q^{i+1}, \ldots, q^{i+1}}_{r-1}
\end{array} ;, \frac{b}{a_{1} \cdots a_{r+1} q} t\right]-A_{0}^{(j)}\right\}+\delta_{j,-1} .
\end{aligned}
$$

We explain the notation appearing in the above theorem. First, $\delta_{i, j}$ is the Kronecker delta defined by $\delta_{i, j}=1$ for $i=j$ and 0 otherwise. Let $\alpha_{1}, \alpha_{2}, \ldots, \alpha_{r+1}$ be variables determined by

$$
\left\{\begin{array}{l}
\alpha_{1}+\alpha_{2}+\cdots+\alpha_{r+1}=x_{2}-x_{1}, \\
\sum_{1 \leq i_{1}<\ldots<i_{j} \leq r+1} \alpha_{i_{1}} \cdots \alpha_{i_{j}}=x_{j+1}-x_{1} x_{j}, \quad 2 \leq j \leq r+1,
\end{array}\right.
$$

and let

$$
b=\frac{q}{1-q_{1} x_{1}}=q\left(1+q_{1} u_{1}\right), a_{i}=\frac{1}{1+q_{1} \alpha_{i}}, \quad i=1, \ldots, r+1 .
$$


Finally, we set

$$
B_{i}=\frac{\left(a_{1}, \ldots, a_{r+1} ; q\right)_{i}}{(b, \underbrace{q, \ldots, q}_{r-1} ; q)_{i}}\left(\frac{b}{a_{1} \ldots a_{r+1} q q_{1}}\right)^{i}, i=0,1, \ldots, r,
$$

and

$$
A_{i}^{(j)}=\sum_{m=i}^{r-1-j}\left(x_{r+2-m}-x_{1} x_{r+1-m}\right) S_{q}(m, i)+x_{1} x_{j+2} S_{q}(r-1-j, i) .
$$

Here $-1 \leq j \leq r-1,0 \leq i \leq r-1-j$ in (3.6).

Using Theorem 3.1, we obtain the main result of this paper, which represents $\Psi_{0}=\Psi_{0}\left(u_{1}, u_{2}, \ldots, u_{r+2}\right)$ by the specializations of the basic hypergeometric function ${ }_{r+1} \phi_{r}$.

Theorem 3.2. We have

$$
\Psi_{0}=\frac{\left[1+q_{1} u_{1}\right]^{2}}{u_{r+2}-u_{1} u_{r+1}}\left\{\sum_{j=0}^{r-1} A_{j} \widetilde{B}_{j r+1} \phi_{r}\left[\begin{array}{c}
a_{1} q^{j}, \ldots, a_{r+1} q^{j} \\
b q^{j}, \underbrace{q^{j+1}, \ldots, q^{j+1}}_{r-1}
\end{array} ; q, \frac{b}{a_{1} \cdots a_{r+1}}\right]-A_{0}\right\} .
$$

In the above, for $0 \leq i \leq r-1$, we define

$$
\widetilde{B}_{i}=\frac{\left(a_{1}, \ldots, a_{r+1} ; q\right)_{i}}{(b, \underbrace{q, \ldots, q}_{r-1} ; q)_{i}}\left(\frac{b}{a_{1} \ldots a_{r+1} q_{1}}\right)^{i}
$$

and

$$
A_{i}=\sum_{m=i}^{r-1} c_{m} S_{q}(m, i)+\frac{u_{1} u_{2}}{\left(1+q_{1} u_{1}\right)^{2}} S_{q}(r-1, i)
$$

with

$$
\begin{aligned}
c_{m}= & \sum_{k=r-m+1}^{r+1}\left[\left(\begin{array}{c}
k-2 \\
r-m
\end{array}\right)+\frac{q_{1} u_{1}}{1+q_{1} u_{1}}\left(\begin{array}{c}
k-2 \\
r-m-1
\end{array}\right)\right]\left(-q_{1}\right)^{k-r+m-2} \\
& \cdot\left(\frac{u_{k}}{1+q_{1} u_{1}}-u_{1}^{k-r-2} u_{r+2}+\frac{q_{1} u_{k+1}}{1+q_{1} u_{1}}\right) .
\end{aligned}
$$

We remark that by taking the limit $q \rightarrow 1$ in Theorem 3.2, we indeed come back to the main result of 4 . Moreover one can get a similar formula for any $\Psi_{j}$.

To end this subsection, we list two computations.

Examples. (1) For $r=1$, we have

$$
\Psi_{0}\left(u_{1}, u_{2}, u_{3}\right)=\frac{u_{3}}{u_{3}-u_{1} u_{2}}\left\{{ }_{2} \phi_{1}\left[\begin{array}{c}
a_{1}, a_{2} \\
b
\end{array} ; q, \frac{b}{a_{1} a_{2}}\right]-1\right\},
$$

where

$$
b=q\left[1+(1-q) u_{1}\right]
$$

and

$$
\left\{\begin{array}{l}
a_{1}+a_{2}=\frac{2+(1-q)\left(u_{1}+u_{2}\right)+(1-q)^{2}\left(u_{1} u_{2}-u_{3}\right)}{1+(1-q) u_{2}} \\
a_{1} a_{2}=\frac{1+(1-q) u_{1}}{1+(1-q) u_{2}}
\end{array}\right.
$$


By using Heine's $q$-analogue of Gauss' summation formula, we obtain a $q$-analogue of Ohno-Zagier's relation deduced by Okuda and Takeyama in [6].

(2) For $r=2$, we have

$$
\begin{aligned}
\Psi_{0}\left(u_{1}, u_{2}, u_{3}, u_{4}\right)= & \frac{q\left[u_{3}+(1-q)\left(u_{1} u_{3}-u_{4}\right)\right]}{1-q u_{1}}{ }_{3} \phi_{2}\left[\begin{array}{c}
a_{1} q, a_{2} q, a_{3} q \\
b q, q^{2}
\end{array} ; q, \frac{b}{a_{1} a_{2} a_{3}}\right] \\
& +{ }_{3} \phi_{2}\left[\begin{array}{c}
a_{1}, a_{2}, a_{3} \\
b, q
\end{array} ;, \frac{b}{a_{1} a_{2} a_{3}}\right]-1,
\end{aligned}
$$

where

$$
b=q\left[1+(1-q) u_{1}\right]
$$

and

$$
\left\{\begin{array}{l}
a_{1}+a_{2}+a_{3}=\frac{3+(1-q)\left(u_{1}+2 u_{2}\right)+(1-q)^{2}\left(u_{1} u_{2}-u_{3}\right)}{1+(1-q) u_{2}}, \\
a_{1} a_{2}+a_{2} a_{3}+a_{3} a_{1}=\frac{3+(1-q)\left(2 u_{1}+u_{2}\right)+(1-q)^{2}\left(u_{1} u_{2}-u_{3}\right)+(1-q)^{3}\left(u_{4}-u_{1} u_{3}\right)}{1+(1-q) u_{2}}, \\
a_{1} a_{2} a_{3}=\frac{1+(1-q) u_{1}}{1+(1-q) u_{2}} .
\end{array}\right.
$$

3.2. Sum of multiple $q$-polylogarithms. We prove Theorem 3.1 in this subsection. The multiple $q$-polylogarithms satisfy the following relations:

$$
\mathcal{D}_{q} \operatorname{Li}_{k_{1}, \ldots, k_{n}}[t]= \begin{cases}\frac{1}{t} \operatorname{Li}_{k_{1}-1, k_{2}, \ldots, k_{n}}[t], & \text { if } k_{1} \geq 2, \\ \frac{1}{1-t} \operatorname{Li}_{k_{2}, \ldots, k_{n}}[t], & \text { if } k_{1}=1 .\end{cases}
$$

Since the formula (3.10) is the same as that of the differential operator $\frac{d}{d t}$ operating on the multiple polylogarithms (of one variable) and recalling that $\Theta_{q}=t \mathcal{D}_{q}$, we can get the following proposition after the same argument as in 4 .

Proposition 3.3. Let $y_{0}=\Phi_{r-1}$ and $y_{j}=\Phi_{r-1-j}-\Phi_{r-j}, j=1,2, \ldots, r$. We have

(1):

$$
y_{j}=\frac{x_{r+2-j}}{x_{r+2}} \Theta_{q}^{j} y_{0}-\frac{x_{1} x_{r+2-j}}{x_{r+2}} \Theta_{q}^{j-1} y_{0}+\delta_{j, r}, j=1, \ldots, r
$$

(2): $y_{0}$ satisfies the following q-difference equation:

$$
\left\{\Theta_{q}^{r}\left(\Theta_{q}-x_{1}\right)-t\left[\Theta_{q}^{r+1}+\left(x_{2}-x_{1}\right) \Theta_{q}^{r}+\sum_{j=0}^{r-1}\left(x_{r+2-j}-x_{1} x_{r+1-j}\right) \Theta_{q}^{j}\right]\right\} y_{0}=t x_{r+2} .
$$

To prove Theorem 3.1, we prepare the following lemma.

Lemma 3.4. Let $m$ be a positive integer, and $p_{i}(t)=c_{i 0}+c_{i 1} t \in \mathbb{C}[t]$ be a degree at most 1 polynomial for $i=0,1, \ldots, m$. Assume that $\sum_{i=0}^{m}[n]^{i} c_{m-i, 0} \neq 0$ for any positive integer $n$. Then the initial problem

$$
\left\{\begin{array}{l}
\left\{p_{0}(t) \Theta_{q}^{m}+p_{1}(t) \Theta_{q}^{m-1}+\cdots+p_{m-1}(t) \Theta_{q}+p_{m}(t)\right\} y=0 \\
y(0)=0
\end{array}\right.
$$

has a unique holomorphic solution $y(t)=0$ for $|t|<1$. 
Proof. It is sufficient to prove the uniqueness. Assume that $y(t)$ is a solution. Then we set $y(t)=\sum_{n=1}^{\infty} a_{n} t^{n}$. By the $q$-difference equation satisfied by $y(t)$, we get

$$
\sum_{n=1}^{\infty}\left(\sum_{i=0}^{m}[n]^{i} c_{m-i, 0}\right) a_{n} t^{n}+\sum_{n=2}^{\infty}\left(\sum_{i=0}^{m}[n-1]^{i} c_{m-i, 1}\right) a_{n-1} t^{n}=0 .
$$

Hence $a_{n}=0$ for any $n \geq 1$.

Now we come to the proof of Theorem 3.1. For $j=r-1$, since $B_{0}=1$ and $A_{0}^{(r-1)}=\left(x_{r+2}-x_{1} x_{r+1}\right) S_{q}(0,0)+x_{1} x_{r+1} S_{q}(0,0)=x_{r+2}$, it is enough to prove that

$$
\Phi_{r-1}=\frac{x_{r+2}}{x_{r+2}-x_{1} x_{r+1}}\left\{r+1 \phi_{r}\left[\begin{array}{c}
a_{1}, \ldots, a_{r+1} \\
b, \underbrace{q, \ldots, q}_{r-1}
\end{array} ; q, \frac{b}{a_{1} \cdots a_{r+1} q} t\right]-1\right\},
$$

which follows from Lemma 2.3. Lemma 3.4, Proposition 3.3 (2) and $\Phi_{r-1}(0)=0$. The general case is a consequence of (3.11), (2.3), (2.2) and the result for $j=$ $r-1$.

3.3. Sum of multiple $q$-zeta values. We prove the main theorem (Theorem 3.2) in this subsection. For an admissible sequence $\mathbf{k}=\left(k_{1}, k_{2}, \ldots, k_{n}\right)$, it is known that the specialization of multiple polylogarithms at 1 is just $\zeta(\mathbf{k})$. So in [4, we only need to set $t=1$ in $\Phi_{0}$ to obtain the generating function of sums of multiple zeta values, while the situation here is more complicated. From [6], we have

$$
\begin{gathered}
\operatorname{Li}_{\mathbf{k}}[q]=\sum_{a_{1}=2}^{k_{1}} \sum_{a_{2}=1}^{k_{2}} \ldots \sum_{a_{n}=1}^{k_{n}}\left(\begin{array}{l}
k_{1}-2 \\
a_{1}-2
\end{array}\right)\left\{\prod_{j=2}^{n}\left(\begin{array}{c}
k_{j}-1 \\
a_{j}-1
\end{array}\right)\right\} \\
\cdot(1-q)^{\sum_{j=1}^{n}\left(k_{j}-a_{j}\right)} \zeta\left[a_{1}, \ldots, a_{n}\right] .
\end{gathered}
$$

Thus we need all the $\Phi_{j}$ 's and some matrix computations.

In this subsection, for any $j$ with $0 \leq j \leq r-1$, we abbreviate $\Phi_{j}\left(x_{1}, \ldots, x_{r+2} ; q\right)$ as $\Phi_{j}(q)$ and $\Psi_{j}\left(u_{1}, \ldots, u_{r+2}\right)$ as $\Psi_{j}$. We set

$$
Y_{l}=\sum_{i=l}^{r}\left(\begin{array}{l}
i-2 \\
l-2
\end{array}\right)\left(q_{1} x_{1}\right)^{i-l} \frac{x_{1}^{r+1-i} x_{i+1}-x_{r+2}}{x_{r+2}}+\left(1-q_{1} x_{1}\right)^{1-l}, l=2, \ldots, r,
$$

and

$$
Z_{l j}=\sum_{i=l}^{j}\left(\begin{array}{l}
i-2 \\
l-2
\end{array}\right)\left(q_{1} x_{1}\right)^{i-l} \frac{x_{1}^{r+1-i} x_{i+1}}{x_{r+2}}, 2 \leq l \leq j \leq r .
$$

Then using (3.12), we obtain the following lemma. 
Lemma 3.5. Let $A$ and $D$ be two $r \times r$ matrices defined by

$$
A=\left(\begin{array}{cccccc}
Y_{2} & Y_{3} & Y_{4} & \cdots & Y_{r} & 1 \\
Y_{2}-Z_{22} & Y_{3} & Y_{4} & \cdots & Y_{r} & 1 \\
Y_{2}-Z_{23} & Y_{3}-Z_{33} & Y_{4} & \cdots & Y_{r} & 1 \\
\vdots & \vdots & \vdots & \ddots & \vdots & \vdots \\
Y_{2}-Z_{2, r-1} & Y_{3}-Z_{3, r-1} & Y_{4}-Z_{4, r-1} & \cdots & Y_{r} & 1 \\
Y_{2}-Z_{2 r} & Y_{3}-Z_{3 r} & Y_{4}-Z_{4 r} & \cdots & Y_{r}-Z_{r r} & 1
\end{array}\right)
$$

and

$$
D=\operatorname{diag}\left(X_{2}^{-1}, X_{3}^{-1}, \ldots, X_{r}^{-1}, 1-q_{1} x_{1}\right) .
$$

We have

$$
\left(\begin{array}{c}
\Phi_{0}(q) \\
\Phi_{1}(q) \\
\vdots \\
\Phi_{r-1}(q)
\end{array}\right)=A D\left(\begin{array}{c}
\Psi_{0}-\Psi_{1} \\
\Psi_{1}-\Psi_{2} \\
\vdots \\
\Psi_{r-2}-\Psi_{r-1} \\
\Psi_{r-1}
\end{array}\right)
$$

Proof. Using (3.12) and changing the order of the sums, we get

$$
\begin{aligned}
& \Phi_{j}(q)= \\
& \sum_{k, n, h_{1}, \ldots, h_{r} \geq 0} \sum_{\left(a_{1}, \ldots, a_{n}\right) \in I_{0}\left(k, n, h_{1}, \ldots, h_{r}\right)} \sum_{k_{i}^{\prime} \geq a_{i}, i=1, \ldots, n, k_{1}^{\prime} \geq j+2}\left(\begin{array}{c}
k_{1}^{\prime}-2 \\
a_{1}-2
\end{array}\right) \prod_{l=2}^{n}\left(\begin{array}{c}
k_{l}^{\prime}-1 \\
a_{l}-1
\end{array}\right) \\
& \cdot q_{1}^{\sum_{l=1}^{n}\left(k_{l}^{\prime}-a_{l}\right)} x_{1}^{k^{\prime}-n-\sum_{l=1}^{r} h_{l}^{\prime}} x_{2}^{n-h_{1}^{\prime}} x_{3}^{h_{1}^{\prime}-h_{2}^{\prime}} \ldots x_{r+1}^{h_{r-1}^{\prime}-h_{r}^{\prime}} x_{r+2}^{h_{r}^{\prime} \zeta\left[a_{1}, \ldots, a_{n}\right],}
\end{aligned}
$$

where $k^{\prime}, h_{1}^{\prime}, \ldots, h_{r}^{\prime}$ are nonnegative integers such that $\left(k_{1}^{\prime}, \ldots, k_{n}^{\prime}\right) \in I_{j}\left(k^{\prime}, n, h_{1}^{\prime}\right.$, $\left.\ldots, h_{r}^{\prime}\right)$. Since

$$
\begin{aligned}
& x_{1}^{k^{\prime}-n-\sum_{j=1}^{r} h_{j}^{\prime}} x_{2}^{n-h_{1}^{\prime}} x_{3}^{h_{1}^{\prime}-h_{2}^{\prime}} \cdots x_{r+1}^{h_{r-1}^{\prime}-h_{r}^{\prime}} x_{r+2}^{h_{r}^{\prime}} \\
& =\left(\frac{x_{r+2}}{x_{1}^{r+1}}\right)^{n} \prod_{m=1}^{n} x_{1}^{k_{m}^{\prime}}\left(\frac{x_{1}^{r} x_{2}}{x_{r+2}}\right)^{\delta_{k_{m}^{\prime}, 1}}\left(\frac{x_{1}^{r-1} x_{3}}{x_{r+2}}\right)^{\delta_{k_{m}^{\prime}, 2}} \cdots\left(\frac{x_{1} x_{r+1}}{x_{r+2}}\right)^{\delta_{k_{m}^{\prime}, r}},
\end{aligned}
$$

we have

$$
\sum_{k, n, h_{1}, \ldots, h_{r} \geq 0} \sum_{\left(a_{1}, \ldots, a_{n}\right) \in I_{0}\left(k, n, h_{1}, \ldots, h_{r}\right)} S^{(j)}\left(a_{1}, \ldots, a_{n}\right) x_{1}^{k}\left(\frac{x_{r+2}}{x_{1}^{r+1}}\right)^{n} \zeta\left[a_{1}, \ldots, a_{n}\right],
$$

where for any fixed $\left(a_{1}, \ldots, a_{n}\right) \in I_{0}\left(k, n, h_{1}, \ldots, h_{r}\right)$, we set

$$
\begin{gathered}
S^{(j)}\left(a_{1}, \ldots, a_{n}\right)=\sum_{k_{i}^{\prime} \geq a_{i}, i=1, \ldots, n, k_{1}^{\prime} \geq j+2}\left(\begin{array}{c}
k_{1}^{\prime}-2 \\
a_{1}-2
\end{array}\right) \prod_{l=2}^{n}\left(\begin{array}{c}
k_{l}^{\prime}-1 \\
a_{l}-1
\end{array}\right)\left(q_{1} x_{1}\right)^{\sum_{l=1}^{n}\left(k_{l}^{\prime}-a_{l}\right)} \\
\cdot \prod_{m=1}^{n}\left(\frac{x_{1}^{r} x_{2}}{x_{r+2}}\right)^{\delta_{k_{m}^{\prime}, 1}}\left(\frac{x_{1}^{r-1} x_{3}}{x_{r+2}}\right)^{\delta_{k_{m}^{\prime}, 2}} \cdots\left(\frac{x_{1} x_{r+1}}{x_{r+2}}\right)^{\delta_{k_{m}^{\prime}, r}} .
\end{gathered}
$$

We compute these terms. Setting

$$
S_{1}^{(j)}=\sum_{i \geq a_{1}, i \geq j+2}\left(\begin{array}{c}
i-2 \\
a_{1}-2
\end{array}\right)\left(q_{1} x_{1}\right)^{i-a_{1}}\left(\frac{x_{1}^{r} x_{2}}{x_{r+2}}\right)^{\delta_{i, 1}}\left(\frac{x_{1}^{r-1} x_{3}}{x_{r+2}}\right)^{\delta_{i, 2}} \cdots\left(\frac{x_{1} x_{r+1}}{x_{r+2}}\right)^{\delta_{i, r}}
$$


and

$$
S_{m}=\sum_{i \geq a_{m}}\left(\begin{array}{c}
i-1 \\
a_{m}-1
\end{array}\right)\left(q_{1} x_{1}\right)^{i-a_{m}}\left(\frac{x_{1}^{r} x_{2}}{x_{r+2}}\right)^{\delta_{i, 1}}\left(\frac{x_{1}^{r-1} x_{3}}{x_{r+2}}\right)^{\delta_{i, 2}} \cdots\left(\frac{x_{1} x_{r+1}}{x_{r+2}}\right)^{\delta_{i, r}}
$$

for $m=2, \ldots, n$, we have $S^{(j)}\left(a_{1}, \ldots, a_{n}\right)=S_{1}^{(j)} S_{2} \cdots S_{n}$. For $a_{1} \geq r+1$, we have

$$
S_{1}^{(j)}=\sum_{i \geq a_{1}}\left(\begin{array}{c}
i-2 \\
a_{1}-2
\end{array}\right)\left(q_{1} x_{1}\right)^{i-a_{1}}=\left(1-q_{1} x_{1}\right)^{1-a_{1}}
$$

for $a_{1}=l$ with $j+2 \leq l \leq r$, we get

$$
S_{1}^{(j)}=\sum_{i=l}^{r}\left(\begin{array}{l}
i-2 \\
l-2
\end{array}\right)\left(q_{1} x_{1}\right)^{i-l} \frac{x_{1}^{r+1-i} x_{i+1}}{x_{r+2}}+\sum_{i \geq r+1}\left(\begin{array}{l}
i-2 \\
l-2
\end{array}\right)\left(q_{1} x_{1}\right)^{i-l}=Y_{l} ;
$$

and for $a_{1}=l$ with $2 \leq l \leq j+1$, we have $S_{1}^{(j)}=Y_{l}-Z_{l, j+1}$. For $S_{m}$, if $a_{m}=l$ with $1 \leq l \leq r$, then we have $S_{m}=X_{l}$; and if $a_{m} \geq r+1$, we get $S_{m}=\left(1-q_{1} x_{1}\right)^{-a_{m}}$. Hence if we set $\widetilde{X}_{l}=X_{l}\left(1-q_{1} x_{1}\right)^{l}$ for $l=1, \ldots, r$, then for $a_{1} \geq r+1$, we have

$$
\begin{aligned}
S^{(j)}\left(a_{1}, \ldots, a_{n}\right)= & \left(1-q_{1} x_{1}\right)^{1-a_{1}} X_{1}^{n-h_{1}} X_{2}^{h_{1}-h_{2}} \cdots X_{r}^{h_{r-1}-h_{r}} \\
& \cdot \frac{\left(1-q_{1} x_{1}\right)^{-\sum_{l=2}^{n} a_{l}}}{\left(1-q_{1} x_{1}\right)^{-\left[\left(n-h_{1}\right)+2\left(h_{1}-h_{2}\right)+\cdots+r\left(h_{r-1}-h_{r}\right)\right]}} \\
= & \frac{1-q_{1} x_{1}}{\left(1-q_{1} x_{1}\right)^{k}} \widetilde{X}_{1}^{n-h_{1}} \widetilde{X}_{2}^{h_{1}-h_{2}} \cdots \widetilde{X}_{r}^{h_{r-1}-h_{r}} ;
\end{aligned}
$$

for $a_{1}=l$ with $j+2 \leq l \leq r$, we have

$$
S^{(j)}\left(a_{1}, \ldots, a_{n}\right)=\frac{Y_{l} X_{l}^{-1}}{\left(1-q_{1} x_{1}\right)^{k}} \widetilde{X}_{1}^{n-h_{1}} \widetilde{X}_{2}^{h_{1}-h_{2}} \ldots \widetilde{X}_{r}^{h_{r-1}-h_{r}}
$$

and for $a_{1}=l$ with $2 \leq l \leq j+1$, we get

$$
S^{(j)}\left(a_{1}, \ldots, a_{n}\right)=\frac{\left(Y_{l}-Z_{l, j+1}\right) X_{l}^{-1}}{\left(1-q_{1} x_{1}\right)^{k}} \widetilde{X}_{1}^{n-h_{1}} \widetilde{X}_{2}^{h_{1}-h_{2}} \ldots \widetilde{X}_{r}^{h_{r-1}-h_{r}} .
$$

Now the result follows from the fact that $I_{0}\left(k, n, h_{1}, \ldots, h_{r}\right)$ is the disjoint union

$$
I_{r-1}\left(k, n, h_{1}, \ldots, h_{r}\right) \sqcup \bigsqcup_{i=0}^{r-2}\left(I_{i}\left(k, n, h_{1}, \ldots, h_{r}\right) \backslash I_{i+1}\left(k, n, h_{1}, \ldots, h_{r}\right)\right),
$$

and the formulas for $S^{(j)}\left(a_{1}, \ldots, a_{n}\right)$.

We begin the proof of Theorem 3.2. Theorem 3.1 gives that

$$
\Phi_{j}(q)=\frac{1}{x_{r+2}-x_{1} x_{r+1}}\left\{\sum_{i=0}^{r-1-j} A_{i}^{(j)} \widetilde{B}_{i} f_{i}-A_{0}^{(j)}\right\}
$$


where $f_{i}={ }_{r+1} \phi_{r}\left[\begin{array}{c}a_{1} q^{i}, \ldots, a_{r+1} q^{i} \\ b q^{i}, \underbrace{q^{i+1}, \ldots, q^{i+1}}_{r-1}\end{array} ;, \frac{b}{a_{1} \ldots a_{r+1}}\right]$. Computing the inverse of the matrices $A$ and $D$ in Lemma 3.5, we get

$\Psi_{0}=$

$$
\frac{\left(1+q_{1} u_{1}\right)^{2}}{u_{r+2}-u_{1} u_{r+1}}\left\{\sum_{j=0}^{r-1}\left[A_{j}^{(0)}+x_{r+2} \sum_{i=1}^{r-1}\left(X_{i+1}\left(1-q_{1} x_{1}\right)-Y_{i+1}\right) \frac{c_{i, j+1}}{x_{1}^{r-i}}\right] \widetilde{B}_{j} f_{j}-A_{0}\right\} .
$$

Here we set

$$
c_{i, j+1}=\sum_{m=1}^{\min (i, r-j)}\left(\begin{array}{c}
i-1 \\
m-1
\end{array}\right)\left(-q_{1}\right)^{i-m} a_{m, j+1}
$$

with

$$
a_{i j}= \begin{cases}S_{q}(r-i, j-1)-x_{1} S_{q}(r-1-i, j-1) & \text { if } i+j \leq r+1, \\ 0, & \text { if } i+j>r+1 .\end{cases}
$$

We omit the details of the computations.

Let $\widetilde{A}_{j}=A_{j}^{(0)}+\Sigma_{1}$ with

$$
\Sigma_{1}=x_{r+2} \sum_{i=1}^{r-1}\left(X_{i+1}\left(1-q_{1} x_{1}\right)-Y_{i+1}\right) \frac{c_{i, j+1}}{x_{1}^{r-i}} .
$$

Then we only need to prove that $\widetilde{A}_{j}=A_{j}$ for any $0 \leq j \leq r-1$. In the rest of the proof we fix one $j$ with $0 \leq j \leq r-1$.

We have that for any $2 \leq l \leq r$,

$$
X_{l}\left(1-q_{1} x_{1}\right)-Y_{l}=\frac{u_{1}^{r+1-l}\left(1+q_{1} u_{1}\right)^{l-1}}{u_{r+2}} \sum_{k=l+1}^{r+1}\left(-q_{1}\right)^{k-l}\left(u_{1} u_{k}-u_{k+1}\right),
$$

which induces

$$
\Sigma_{1}=\frac{1}{1+q_{1} u_{1}} \sum_{i=2}^{r} \sum_{k=i+1}^{r+1} \sum_{m=1}^{\min (i-1, r-j)}\left(\begin{array}{c}
i-2 \\
m-1
\end{array}\right)\left(-q_{1}\right)^{k-m-1}\left(u_{1} u_{k}-u_{k+1}\right) a_{m, j+1} .
$$

Changing the order of the sums, we get

$$
\Sigma_{1}=\frac{1}{1+q_{1} u_{1}} \sum_{m=1}^{r-j} \sum_{k=m+2}^{r+1}\left(\begin{array}{c}
k-2 \\
m
\end{array}\right)\left(-q_{1}\right)^{k-m-1}\left(u_{1} u_{k}-u_{k+1}\right) a_{m, j+1} .
$$

In the above formula, $a_{m, j+1}=S_{q}(r-m, j)-x_{1} S_{q}(r-m-1, j)$, which implies that

$$
\begin{aligned}
& \Sigma_{1}=\sum_{m=j}^{r-1}\left\{\sum_{k=r-m+1}^{r+1}\left[\left(\begin{array}{c}
k-2 \\
r-m
\end{array}\right)+\frac{q_{1} u_{1}}{1+q_{1} u_{1}}\left(\begin{array}{c}
k-2 \\
r-m-1
\end{array}\right)\right]\right. \\
&\left.\quad \times \frac{\left(-q_{1}\right)^{k-r+m-1}}{1+q_{1} u_{1}}\left(u_{1} u_{k}-u_{k+1}\right)\right\} S_{q}(m, j) \\
&+ {\left[\frac{u_{1}}{\left(1+q_{1} u_{1}\right)^{2}} \sum_{k=2}^{r+1}\left(-q_{1}\right)^{k-1}\left(u_{1} u_{k}-u_{k+1}\right)\right] S_{q}(r-1, j) . }
\end{aligned}
$$


By the definition of $A_{j}^{(0)}$, and after some computations, we obtain

$$
\begin{aligned}
A_{j}^{(0)}=\sum_{m=j}^{r-1}\left\{\sum_{k=r-m+1}^{r+1}\left[\left(\begin{array}{c}
k-2 \\
r-m
\end{array}\right)+\frac{q_{1} u_{1}}{1+q_{1} u_{1}}\left(\begin{array}{c}
k-2 \\
r-m-1
\end{array}\right)\right]\right. \\
\left.\cdot\left(-q_{1}\right)^{k-r+m-2} u_{1}^{k-r-2}\left(u_{1}^{r-k+2} u_{k}-u_{r+2}\right)\right\} S_{q}(m, j) \\
+\left[\frac{u_{1}}{1+q_{1} u_{1}} \sum_{k=2}^{r+1}\left(-q_{1}\right)^{k-2} u_{k}+\frac{\left(-q_{1}\right)^{r} u_{1} u_{r+2}}{\left(1+q_{1} u_{1}\right)^{2}}\right] S_{q}(r-1, j) .
\end{aligned}
$$

Finally, we get

$$
\widetilde{A}_{j}=A_{j}^{(0)}+\Sigma_{1}=\sum_{m=j}^{r-1} \tilde{c}_{m} S_{q}(m, j)+c S_{q}(r-1, j),
$$

where

$$
\begin{aligned}
& \tilde{c}_{m}=\sum_{k=r-m+1}^{r+1}\left[\left(\begin{array}{c}
k-2 \\
r-m
\end{array}\right)+\frac{q_{1} u_{1}}{1+q_{1} u_{1}}\left(\begin{array}{c}
k-2 \\
r-m-1
\end{array}\right)\right] \\
& \text { · }\left(-q_{1}\right)^{k-r+m-2} u_{1}^{k-r-2}\left(u_{1}^{r-k+2} u_{k}-u_{r+2}\right) \\
& +\sum_{k=r-m+1}^{r+1}\left[\left(\begin{array}{c}
k-2 \\
r-m
\end{array}\right)+\frac{q_{1} u_{1}}{1+q_{1} u_{1}}\left(\begin{array}{c}
k-2 \\
r-m-1
\end{array}\right)\right] \frac{\left(-q_{1}\right)^{k-r+m-1}}{1+q_{1} u_{1}}\left(u_{1} u_{k}-u_{k+1}\right)
\end{aligned}
$$

and

$c=\frac{u_{1}}{1+q_{1} u_{1}} \sum_{k=2}^{r+1}\left(-q_{1}\right)^{k-2} u_{k}+\frac{\left(-q_{1}\right)^{r} u_{1} u_{r+2}}{\left(1+q_{1} u_{1}\right)^{2}}+\frac{u_{1}}{\left(1+q_{1} u_{1}\right)^{2}} \sum_{k=2}^{r+1}\left(-q_{1}\right)^{k-1}\left(u_{1} u_{k}-u_{k+1}\right)$.

It is trivial to find that $c=\frac{u_{1} u_{2}}{\left(1+q_{1} u_{1}\right)^{2}}$, so what we need to show is $\tilde{c}_{m}=c_{m}$. However this is clear. We have finished the proof of Theorem 3.2

\section{REFERENCES}

[1] D. M. Bradley, Multiple q-zeta values, J. Algebra, 283(2005), 752-798. MR2111222 (2006f:11106)

[2] G. Gasper and M. Rahman, Basic hypergeometric series, second edition, Cambridge University Press, Cambridge (England) and New York, 2004. MR.2128719 (2006d:33028)

[3] H. W. Gould, The $q$-Stirling numbers of the first and second kinds, Duke Math. J., 28(1961), 281-289. MR0122759 (23:A99)

[4] Z. Li, Sum of multiple zeta values of fixed weight, depth and $i$-height, Math. Z., 258(2008), 133-142. MR 2350039 (2008j:11118)

[5] Y. Ohno and D. Zagier, Multiple zeta values of fixed weight, depth and height, Indag. Math. (N.S.), 12(2001), 483-487. MR.1908876 (2003e:11094)

[6] J. Okuda and Y. Takeyama, On relations for the multiple $q$-zeta values, The Ramanujan Journal, 14(2007), 379-387. MR2357443 (2008j:11011) 
[7] M. Wachs and D. White, $p, q$-Stirling numbers and set partition statistics, J. Combin. Theory Ser. A, 56(1991), 27-46. MR.1082841 (92b:05004)

[8] J. Zhao, multiple $q$-zeta functions and multiple $q$-polylogarithms, The Ramanujan Journal, 14 (2007), 189-221. MR2341851 (2008h:11095)

Graduate School of Mathematical Science, University of Tokyo, 3-8-1 Komaba, Meguro, Tokyo, 153-8914, Japan

Current address: Department of Mathematics, Tongji University, Shanghai 200092, People's Republic of China

E-mail address: lizhmath@gmail.com 